\title{
A Cloud Monitoring System for Remote Sites
}

\author{
R. W. Clay, N. R. Wild, D. J. Bird, B. R. Dawson, \\ M. Johnston, R. Patrick and A. Sewell \\ Department of Physics and Mathematical Physics, \\ University of Adelaide, SA 5005, Australia \\ rclay@physics.adelaide.edu.au
}

Received 1998 February 16, accepted 1998 August 27

\begin{abstract}
A cloud monitor has been developed for use with cosmic ray air shower fluorescence detectors, the High Resolution Fly's Eye and the Pierre Auger Observatory. This is based on an infrared thermopile device which, unlike previous such monitors, requires no moving chopper and is suitable for unattended operation over long periods of time.
\end{abstract}

Keywords: instrumentation: detectors

\section{Introduction}

Many astronomical observations require information on the cloud cover at the time of the observation (either day or night). Such information has not been simple or cheap to obtain or has been unreliable in the case of visual estimates made by observers. We were presented with a particular such requirement in connection with atmospheric fluorescence detectors which are currently used for the detection and analysis of the highest energy cosmic ray showers. Such detectors are currently employed by the High Resolution Fly's Eye (HiRes) (Bird et al. 1995) and will be used by the Pierre Auger Observatory (Boratov et al. 1997). What was required was a cloud detector with a substantial field of view which could be used to determine whether or not there was cloud in the viewing direction of a mirror collecting atmospheric fluorescence light. Such a cloud detector might be used with a limited number of others to cover the complete sky. The characteristic angular field of view of a detector would be of the order of twenty degrees.

Fluorescence detectors such as HiRes view the whole sky with many photomultiplier pixels which are sensitive to the nitrogen fluorescence light produced in the atmosphere by the passage of a cosmic ray cascade. Cascade images are thus obtained for distances up to some tens of kilometres. The analysis of the data requires a knowledge of whether or not any particular part of a cascade track is obscured by clouds. The fluorescence detectors operate continuously on nominally clear moonless nights and many mirrors may be involved, possibly at remote sites. The cloud detectors must therefore be robust, requiring no attention for perhaps years on end, and they must draw little current since they may be at a site which is solar powered with limited current resources.

(C) Astronomical Society of Australia 1998
We have taken an idea for a cloud detector for automated telescopes by Ashley \& Jurcevic (1991) using an infrared pyroelectric element and developed it into a device based on a thermopile element which requires no mechanical chopping and draws little current.

\section{Thermopile Cloud Detector}

Sloan, Shaw \& Williams (1955) have provided data which show that infrared measurements of the sky at wavelengths above $5 \mu \mathrm{m}$ (in particular between 8 and $14 \mu \mathrm{m}$ ) are sensitive to the presence of clouds. The presence of clouds produces an enhanced signal (which corresponds to an approximate black body spectrum at about ground temperature) above that from the clear sky. There is also an effect of atmospheric humidity which can produce enhanced signals particularly at low elevations. Inexpensive infrared sensors are now available which make allsky or limited direction cloud monitoring possible without detector cooling systems and thus have low current drain with simplified design constraints.

The cloud detector described by Ashley \& Jurcevic (1991) satisfied many of the requirements for a monitoring detector, but from our point of view had two disadvantages which were (a) its requirement for mechanical chopping and (b) its use of a mirror. These aspects would limit its long term reliability in a remote desert environment. The requirement for chopping is inherent in the pyroelectric infrared element. Such an element responds to an infrared signal with adequate sensitivity but returns to its DC level with a time scale of the order of a second. Thus chopping between the sky and a comparison with a frequency of the order of $1 \mathrm{~Hz}$ is required. The concept for the use of a cloud detector with the Auger array is that detectors may be attached to many of the 3000 cosmic ray detectors at each site. 


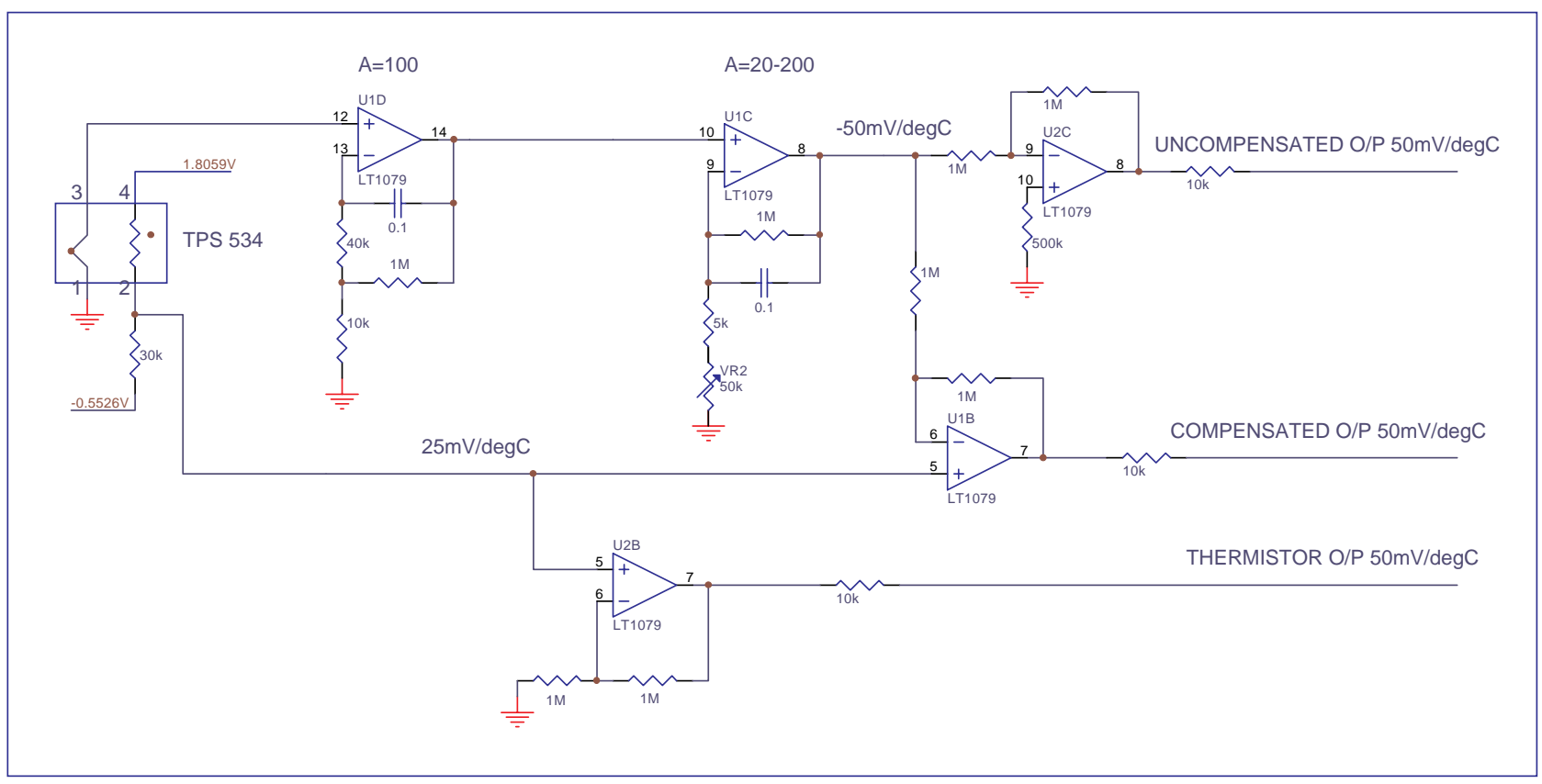

Figure 1-Circuit schematic of the cloud detector. A thermistor measures the internal temperature of the detector canister and this is used to provide a reference for the thermopile element. The compensated output thus corresponds to the temperature in the detector field of view.

They would operate remotely and have no daytime weather protection. For this purpose, a mirror is not likely to be suitable due to deterioration from dust. A mirror or an infrared fresnel lens might well be suitable for other astronomical applications.

Thermoelectric sensors available now have similar sensitivity to the pyroelectric detectors but will maintain an output voltage proportional to the temperature difference between the field of view and a local comparison within the detector canister. We have now based our cloud detectors on such sensors. We monitor the detector canister temperature with an internal thermistor which allows us to derive an analog signal corresponding to the temperature over the field of view or to later calculate that value from ADC measurements of the output signals. With the data presented below, the correction for the canister temperature is substantial. In situations where the detector is deployed only at night or, as will be the case for the Auger array, it will be shielded from the sun and attached to a massive body with a long thermal time constant, the correction will be smaller and more straightforward. We have operated detectors of this sort for up to six months and have found no problems due to long term drifts. The sensor elements are sufficiently sensitive that we do not require a mirror for monitoring the presence of visible clouds. It is possible that sub-visual cirrus may be detectable with the aid of a mirror as suggested by Ashley \& Jurcevic but this was not our present purpose.

We define a field of view by mechanical collimation. In most cases, this was achieved by mounting the sensor element behind a small aluminium tube made to have a diameter of $8.2 \mathrm{~mm}$ and a length of $14.5 \mathrm{~mm}$. With the very small detector sensitive area, this results in a field of view with a full angle of about $30^{\circ}$. We have made detectors with fields of view down to one tenth of this value with the use of infrared fresnel lenses such as are used in security intruder sensors.

We chose to use an EG\&G Heimann type TPS 534 thermopile detector which has a sensitive area of $1.2 \times 1.2 \mathrm{~mm}^{2}$, a responsivity of $42 \mathrm{~V} / \mathrm{W}$ and a filter which passes wavelengths above $5.5 \mu \mathrm{m}$. The sensor itself is modest in cost and a complete cloud detector pixel can be built for below AUS\$200 in component costs (at one off prices). This includes using low-power precision components with low drifts and offsets, required for an analog correction to allow for the canister temperature.

The circuitry (Figure 1) was designed to have low current drain and can operate continuously for well over a month using a $9 \mathrm{~V}$ alkaline battery (supply current below $400 \mu \mathrm{A}$ ).

\section{Sensitivity to Cloud}

The detector was tested for sensitivity to cloud coverage on a day which had rapid changes in the cumulus coverage. Sky photographs were taken whilst the device was operating and the fraction of cloud in the field of view was related to the output signal. This was found to be a linear relationship (see Figure 2).

For the purpose of the following figures, the data are presented at one hour resolution. However, the data were logged at one minute intervals. The cloud 


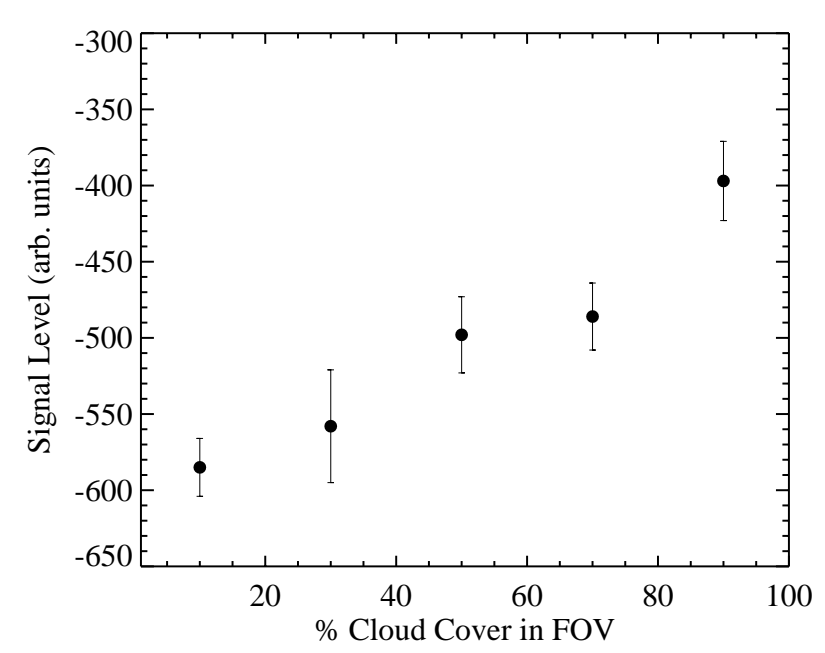

Figure 2-Relationship between cloud coverage in the field of view and the output of a thermopile cloud detector. The cloud cover (cumulus) was obtained photographically and matched to the detector field of view. monitor field of view had a half angle of $15^{\circ}$. Figure 3 shows the temperature of the detector canister and the compensated detector output derived by adding the canister temperature to the the uncompensated detector output as indicated in the circuit of Figure 1. All the signals are conditioned within the monitor electronics to $50 \mathrm{mV}$ per degree Centigrade. Data taken over 25 December to 7 January 1998 are shown in Figure 3. The sky was predominantly clear over this period. The exceptions were daytime cloud on 26 December, followed by intermittent cloud through the rest of the day and a two day period of cloud beginning in late morning on 1 January. On 5 and 6 January there was a build up of cirrus followed by thick stratocumulus. We believe that the peak early on the morning of 29 December was short-lived cloud. It is evident that the discrimination between a clear sky and cloud in the field of view is straightforward at most times.

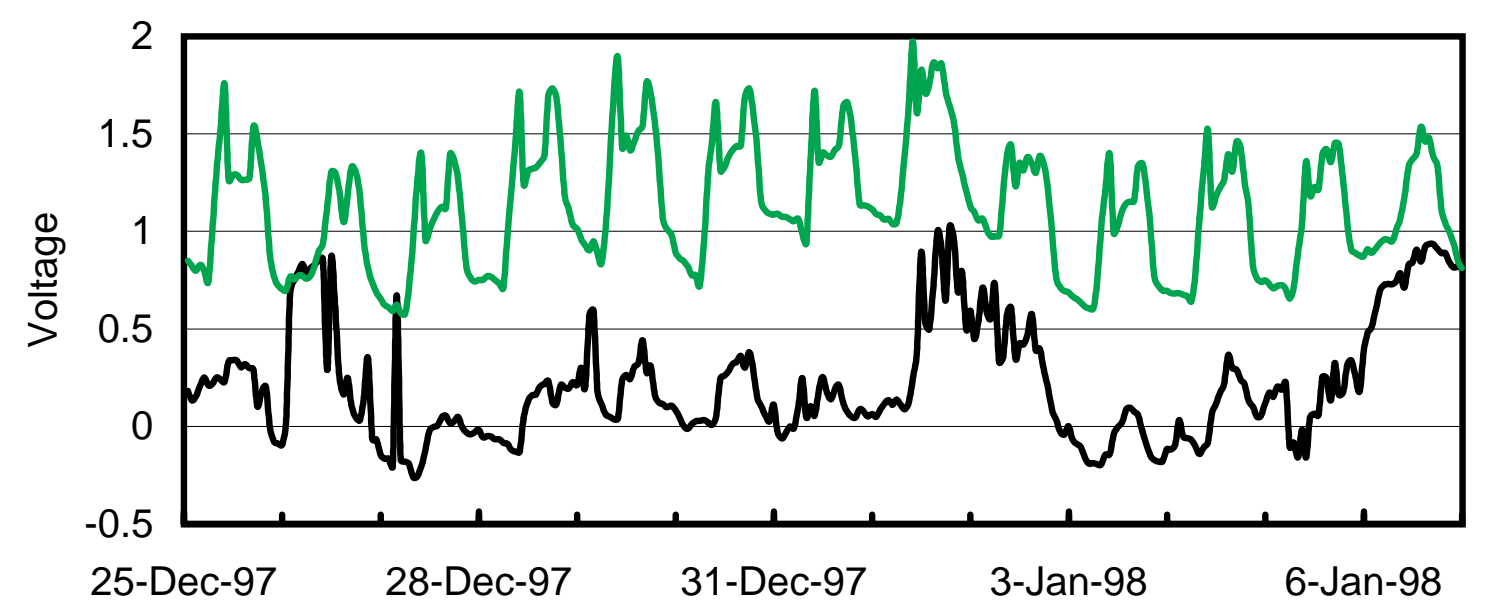

Figure 3-Cloud monitor output over a thirteen day period. The dates indicate the start of the day at midnight. A voltage (upper line) derived from a thermistor gives the detector canister temperature. The lower line gives the compensated output voltage of the sensor element. Both these datasets can be scaled to temperatures with a conversion of $20^{\circ} \mathrm{C}$ per volt.

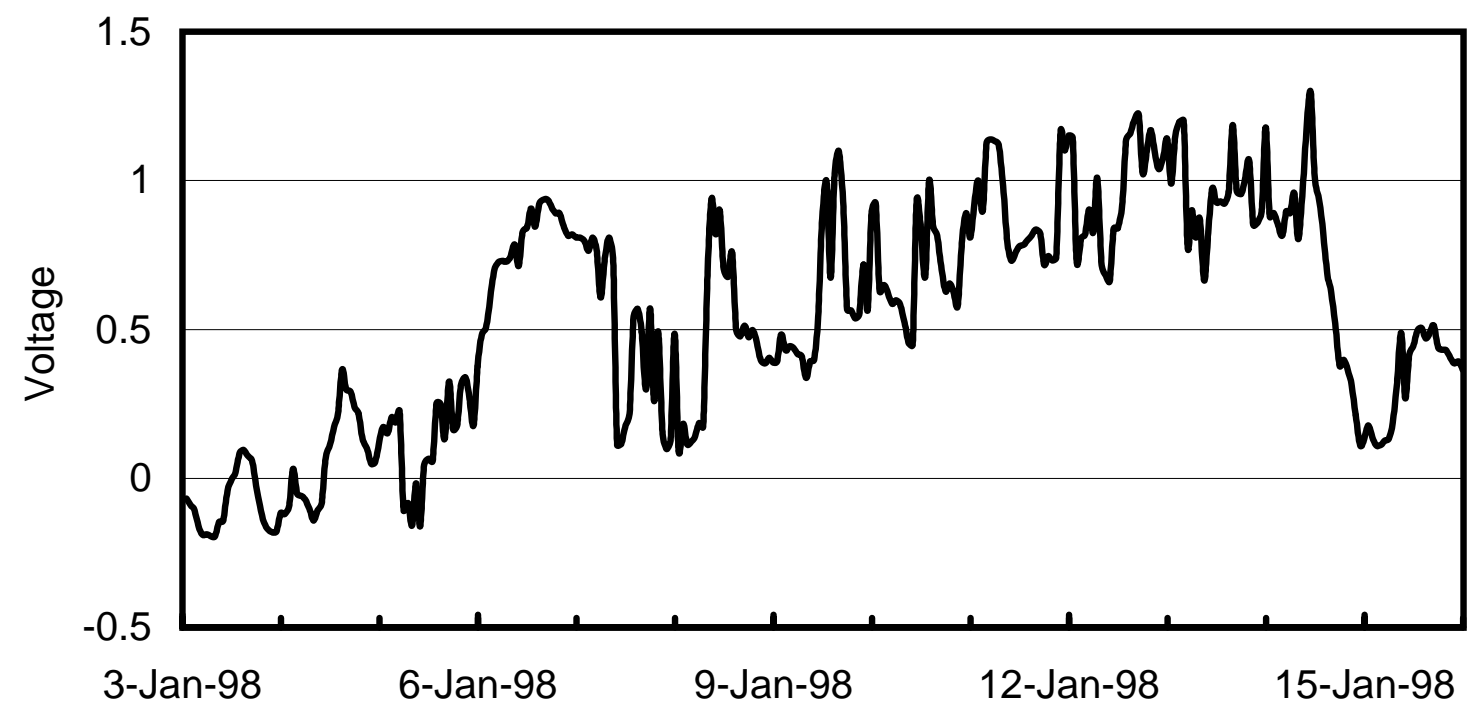

Figure 4-Cloud monitor output over an extended period including a progressive increase in atmospheric moisture prior to the arrival of a weather front arriving at $13 \cdot 00$ on 14 January. January 6 had complete cloud cover. The following days had intermittent cloud (mainly cumulus) except for the night of 8-9 January which was almost clear. 
The monitor viewed towards the south at an elevation of $60^{\circ}$ and was backed by an east-west wall. The result was that the monitor was in direct sunlight in the morning and in the evening, causing considerable temperature variations to occur in the canister at those times (Figure 3). The temperature compensation is not perfect under those conditions but, in terms of defining a cloud-free or cloudy field of view, it remained adequate under difficult circumstances. There is little random noise effect in the data as can be seen from the results over the clear sky period where the signal is lowest. The result is that cloud in the field of view is readily detectable by the setting of an output threshold level.

We have built a second detector which is similar to the first except that it has a window which passes wavelengths above $7 \mu \mathrm{m}$. The agreement in the outputs of the detectors is very good although there are some minor discrepancies from time to time. These may well be related to atmospheric humidity since there is a strong water vapour band between 5 and $8 \mu \mathrm{m}$. However, it is clear that the exact wavelength pass band is not critical to the operation of the cloud monitor.

Sloan, Shaw \& Williams (1955) showed that at times of increased humidity the integrated signal at wavelengths above the water vapour band increases through a general increase at all wavelengths. We have observed this effect as a baseline shift at times of high humidity. The effects of clouds are still clear but it is then necessary to define a 'clear sky' output level at the beginning of an observation period rather than using an absolute level which can be set over long periods at other times. In Adelaide at least, the atmospheric water vapour content varies slowly with a time constant of the order of a day and so a 'clear sky' level which is set at the start of a night will be adequate unless a weather front passes in the observing period. Figure 4 shows an exceptional period in which there was a considerable build-up of atmospheric moisture (from 6 Jan to 14 Jan) from an influx of tropical air, followed by a front which replaced that moist air. The atmosphere became progressively more humid over that period although the effect is probably due to aerosol scattering of water droplets rather than water vapour since it covers a large wavelength range outside the water vapour band. Conditions of such humidity would be rare at the sites selected for the HiRes and Auger projects. We have tested the detector under humid, tropical conditions and it responded to cloud but with a significantly changed baseline, such as was observed in Adelaide in the exceptional January humid period. It certainly appears to be likely to continue to be useful under those conditions, although more testing in the field is warranted to determine what cloud altitude can readily be accessed and whether the baseline is likely to show significant drifts overnight.

\section{Conclusions}

We have constructed cloud monitors based on simple infrared detectors which are sensitive to all visual clouds in their field of view. With compensation for temperature variations in the canister of the infrared element, it is possible to produce an output which can veto cloudy periods with a simple voltage level veto. The cloud detector has no moving components and draws little current. It is thus suitable for remote long term cloud monitoring.

\section{Acknowledgment}

This work was supported by the Australian Research Council.

\section{References}

Ashley, M. C. B., \& Jurcevic, J. S. 1991, PASA, 9, 334

Bird, D. J., et al. 1995, Proc. 24th Int. Cosmic Ray Conference, Rome, ed. N. Iucci, Vol. 3 (University of Rome), p. 504

Boratov, M., et al. 1997, Proc. 25th Int. Cosmic Ray Conference, Durban, ed. M. S. Potgieter, Vol. 5 (Potchefstroomse Universiteit), p. 205

Sloan, R., Shaw, J. H., \& Williams, D. 1955, J. Opt. Soc. Am., 45, 455 